\title{
Sur une nouvelle méthode d'étude de la diffusivité turbulente $\left(^{*}\right)$
}

\author{
L. Petit $(* *)$ \\ Laboratoire d'Hydrodynamique et de Mécanique Physique, E.S.P.C.I. rue Vauquelin, 75005 Paris, France \\ et E. Guyon $(* * *)$ \\ Laboratoire de Physique des Solides, Université Paris-Sud, 91405 Orsay, France
}

(Reçu le 2 avril 1979, accepté le 30 août 1979)

\begin{abstract}
Résumé. - En 1926, L. F. Richardson a proposé l'existence d'une loi de diffusion $D \propto l^{4 / 3}$ pour décrire l'augmentation de la séparation moyenne de paires de particules distantes de $l$ dans un écoulement turbulent. Cette loi peut être étudiée à partir d'une expérience, utilisant la figure de diffraction d'une tache thermique écrite dans un écoulement turbulent, proposée par de Gennes [2]. Nous développons dans ce travail l'analyse de cette approche en montrant d'une part l'existence de solutions simples du problème de Richardson à une dimension et en étudiant la loi d'évolution en temps de la figure de diffraction.
\end{abstract}

\begin{abstract}
In 1926, L. F. Richardson proposed a diffusion law $D(l) \propto l^{4 / 3}$ to describe the increase in the mean separation of pairs of particles separated by a distance $l$ in a turbulent flow. This law can be studied from experimental results, using the diffraction image of a thermal grid established for the turbulent flow, by de Gennes [2]. We develop this analysis, in connection with an experimental approach, by demonstrating, in particular, the existence of simple solutions of the Richardson problem in one dimension, and by studying the time dependence of the diffraction image.
\end{abstract}

1. Introduction. - La mesure directe du champ de gradients de vitesse - la strophométrie - dans un écoulement laminaire ou turbulent présente un intérêt considérable en hydrodynamique; on dispose actuellement d'assez peu de techniques expérimentales qui n'utilisent pas comme intermédiaire la mesure du champ de vitesse lui-même. (Notons cependant des mesures directes utilisant l'alignement partiel de molécules browniennes allongées dans un écoulement ou encore la rotation de molécules sphériques [1].) Dans une lettre récente, de Gennes [2] a suggéré une méthode de mesure des gradients utilisant l'écriture, dans un fluide en écoulement, d'une tache thermique produite par un laser pulsé de forte puissance (LP) incident sur l'écoulement suivie de la lecture de cette tache, au bout d'un temps $\Delta t$ après l'écriture, par un deuxième laser $\left(\mathrm{La}_{2}\right)$ qui en fait une image de diffraction. Les deux caractéristiques essentielles de cette technique sont les suivantes :

(*) Pris en charge en partie par une A.T.P. « Instabilité et Turbulence " du CNRS.

$(* *)$ Fait l'objet d'un travail de recherche d'une thèse de $3^{\mathrm{e}}$ cycle, Orsay (1979).

$\left({ }^{* *}\right)$ Aussi L.H.M.P., E.S.P.C.I., rue Vauquelin, 75005 Paris. i) Les effets de translation de la tache thermique ne sont pas vus dans l'analyse par figure de diffraction, qui est sensible uniquement aux gradients de vitesse dans un écoulement laminaire.

ii) Par ailleurs, en régime turbulent, la figure de diffraction donne accès directement à une donnée essentielle pour l'analyse de la diffusion turbulente la fonction de corrélation à deux points. L'objet de cet article est de donner une description détaillée de cette proposition.

Nous avons mis en place une expérience s'appuyant directement sur la description donnée plus haut; les résultats des premières expériences utilisant un profil d'écoulement laminaire simple (Poiseuille plan) et qui seront décrits indépendamment [3] confirment les propositions du point i).

Le plan de l'article est le suivant. Dans un second chapitre, nous rappellerons quelques résultats essentiels de l'étude de la diffusion de paires de particules en nous appuyant d'une part sur la description des échelles de la turbulence et de l'autre sur l'approche de pionnier et remarquablement intuitive de Richardson [4]. 
Le chapitre 3 s'attache à une résolution de l'équation de diffusion de paires de particules (équation de Richardson) dans un problème à une dimension. Les résultats de ce chapitre sont exploités dans le chapitre 4 dans le cadre de l'expérience de diffraction décrite plus haut. Enfin, un dernier chapitre discute l'extension de ce problème modèle au cas tridimensionnel.

L'analyse qui suit discute le problème d'étalement d'une tache thermique, mais pourrait être étendue au cas de diffusion massique. Cette dernière situation peut être produite expérimentalement à partir du même dispositif expérimental en utilisant un liquide contenant des molécules photochromes excitables par le laser LP (par exemple une solution de rouge de méthyle dont la transition cis-trans peut être excitée à une longueur d'onde $\sim 0,5 \mu[5])$. Le spectre d'absorption dans l'état excité est différent de celui dans l'état au repos et la variation de concentration de molécules excitées est "lue» par le laser $\mathrm{La}_{2}$ essentiellement comme un réseau d'amplitude (c'était un réseau de phase dans le cas thermique). La diffusivité massique étant plus faible que la diffusivité thermique, les résultats que nous discutons sont transposables au cas massique (à condition, bien sûr, que la durée de vie de l'état photochrome excité soit plus longue que celle définie pour la diffusivité dans le réseau, ce qui est facilement réalisée; cette durée de vie est de $10^{-2}$ à $1 \mathrm{~s}$, suivant les solvants, dans le cas du rouge de méthyle).

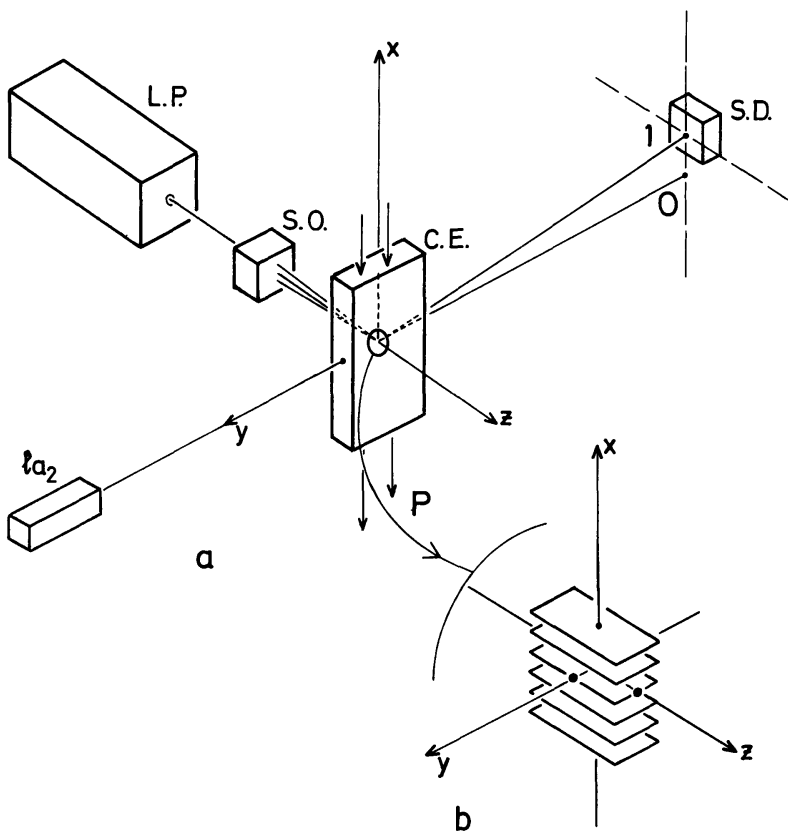

Fig. 1. - Schéma de l'expérience. Un laser pulsé (LP) crée une perturbation de température localisée dans une cellule d'écoulement (CE). L'interposition d'un séparateur de faisceau (SO) permet que cette perturbation soit sous forme d'une grille thermique (b). Un laser de lecture $\left(\mathrm{La}_{2}\right)$ fait de cette grille en écoulement une figure de diffraction lue par le détecteur SD.

[Experimental : a pulsed laser (LP) creates a periodic modulation of temperature (Fig. $1 b$ ) within a flow cell (CE), thanks to an optical interferometric system $(\mathrm{SO})$. A second laser $\left(\mathrm{La}_{2}\right)$ produces a diffraction image of the grating, which is read by the detector (SD).]
Avant de passer au chapitre suivant, nous allons décrire rapidement l'expérience que nous avons mise au point et qui est montrée schématiquement sur la figure 1.

L'impulsion brève $(\sim 20 \mathrm{~ns})$ d'un laser de forte puissance (LP) permet de chauffer instantanément et localement un fluide en écoulement, suivant un profil de température sinusoïdal obtenu à l'aide d'un séparateur optique (SO) (Fig. 1b).

Une cellule d'écoulement (CE) de section rectangulaire permet d'obtenir, suivant sa petite dimension, un profil de vitesse parabolique dont nous déterminons le gradient.

La lumière d'un second laser $\left(\mathrm{La}_{2}\right)$ est diffractée par la perturbation de température, avec un décalage de temps $\Delta t$ réglable entre l'écriture et la lecture. Le gradient local de vitesse est mesuré comme une rotation de la figure de diffraction (matérialisée par les ordres 0 et 1 sur la figure $1 a$ ) par rapport à l'axe vertical, à l'aide du système détecteur (SD) qui est un photomultiplicateur ou une plaque photographique.

2. Diffusion de paires de Richardson. - 2.1 DIFFUSION THERMIQUE. - La diffusion d'une perturbation initiale de température peut se faire de deux façons différentes : d'une part par les mécanismes moléculaires de diffusion thermique en écoulement laminaire, d'autre part par les structures tourbillonnaires fluctuantes de l'écoulement turbulent. Dans ce dernier cas, qui nous intéresse ici, la diffusivité moléculaire n'intervient qu'aux plus petites échelles de l'écoulement turbulent. En effet, d'après les hypothèses de similitude de Kolmogorov [6], si $v$ et $\kappa$ représentent respectivement la viscosité cinématique et la diffusivité thermique du liquide et si $\varepsilon$ est la dissipation d'énergie cinétique par unité de temps et de masse, les microéchelles de Kolmogorov sont

$$
\xi \sim\left(v^{3} / \varepsilon\right)^{1 / 4} \text { et } l^{*} \sim\left(\kappa^{3} / \varepsilon\right)^{1 / 4} .
$$

Leur rapport est donc $\xi / l^{*} \sim(\operatorname{Pr})^{3 / 4}$. Dans nos expériences, le nombre de Prandtl est supérieur à $1(\operatorname{Pr} \sim 7$ pour l'eau). Ainsi, la diffusion de la perturbation de température sera caractérisée complètement par les mécanismes de diffusion turbulente de l'échelle $\mathrm{L}$ (caractérisant les grands tourbillons et définie par la géométrie de l'écoulement) à l'échelle $\xi$. Dans le cas d'un liquide ayant un nombre $\operatorname{Pr}<1$ (un métal liquide), la diffusion thermique moléculaire introduirait une coupure dans l'effet de la cascade à l'échelle $l^{*}$ $(>\xi)$.

2.2 La fonction De Richardson. - Pour que la diffusion d'une perturbation de température puisse être décrite à partir d'une équation de diffusion classique (équation de Fourier), il faudrait que le coefficient de diffusion turbulente soit indépendant de l'échelle du mouvement.

Il est possible de montrer théoriquement [13] qu'une telle approximation (correspondant à une hypothèse 
de coupure dans les équations de diffusion turbulente) est incorrecte. Une solution à l'équation de Fourier stochastique décrivant la diffusion thermique dans un champ de vitesse turbulent s'écrirait sous une forme :

$$
\langle\delta T(\mathbf{k}, t)\rangle=-D(|\mathbf{k}|) k^{2}\langle\delta T(\mathbf{k}, t)\rangle
$$

où $\langle\delta T(\mathbf{k}, T)\rangle$ représente la valeur moyenne de la composante de Fourier de la fluctuation thermique. La diffusivité $D(|\mathbf{k}|)$ diverge pour de faibles vecteurs d'onde $\mathbf{k}$ parce que la fonction de corrélation du champ de vitesse turbulent ne tend pas assez vite vers zéro pour les grandes séparations. En d'autres termes, l'effet de la viscosité sur les grands mouvements est insuffisant pour assurer l'amortissement des fluctuations de $\delta T(\mathbf{r}, t)$ sur des temps et des séparations longues et, par suite, l'existence d'une diffusivité dans ce cas.

Par ailleurs, les expériences de Richardson utilisant la mesure de la variation de la distance de séparation, $l$, entre deux objets, avec le temps montrent que la diffusivité $D$ caractérisant ce processus augmente très rapidement avec $l$; dans le cas d'une diffusion de type moléculaire, cette diffusivité serait égale à 2 fois le coefficient de diffusivité ordinaire pour la diffusion d'une seule particule.

Nous considérons, dans la description modèle qui suit, un système à une dimension caractérisé par une fluctuation de température le long de l'axe $x$ à un instant donné $t, \delta T(x, t)$.

La fonction

$$
q(l, t)=\frac{\int_{-\infty}^{+\infty} \delta T(x, t) \delta T(x+l, t) \mathrm{d} x}{\int_{-\infty}^{+\infty} \delta T(x, t) \mathrm{d} x}
$$

donne la corrélation de la fluctuation de température $\delta T$ pour tous les couples de points séparés d'une distance $l$ sur l'axe. On appellera la fonction $q(l, t)$, qui est la transposée directe de la fonction définie par Richardson pour la diffusion d'un ensemble de particules situées sur un axe, fonction de Richardson pour le problème thermique.

L'intérêt de cette fonction est de prendre en compte directement l'effet de la séparation $l$ de particules qui n'est pas décrite par la fonction $\delta T(x, t)$.

\section{Un exemple :}

Afin de nous familiariser avec la signification de $q(l)$ nous avons choisi une perturbation

$$
\begin{aligned}
& \delta T(x)=\delta T_{0}\left(\exp \left[-\{(x-d) / a\}^{2}\right]\right. \\
&\left.+\exp \left[-\{(x+d) / a\}^{2}\right]\right)
\end{aligned}
$$

correspondant à l'échauffement du liquide par deux faisceaux gaussiens de lumière, de largeur $2 a$ et centrés en $x= \pm d$. La fonction $q(l)$ obtenue s'écrit

$$
\begin{aligned}
q(l)=\left[\delta T_{0} /(2 \sqrt{2})\right] & \left\{2 \exp \left[-\{l /(\sqrt{2} a)\}^{2}\right]+\right. \\
+ & \exp \left[-\{(l-2 d) /(\sqrt{2} a)\}^{2}\right] \\
+ & \left.\exp \left[-\{(l+2 d) /(\sqrt{2} a)\}^{2}\right]\right\} .
\end{aligned}
$$

La variation de $q(l)$ est portée sur la figure 2 avec celle de la fonction $\delta T(x)$. Le pic central de $q(l)$ correspond à la corrélation de tous les couples de points appartenant au même pic de la fonction $\delta T(x)$; il a une amplitude double des deux pics latéraux de même largeur correspondant à des points pris dans les deux pics différents de $\delta T(x)$.
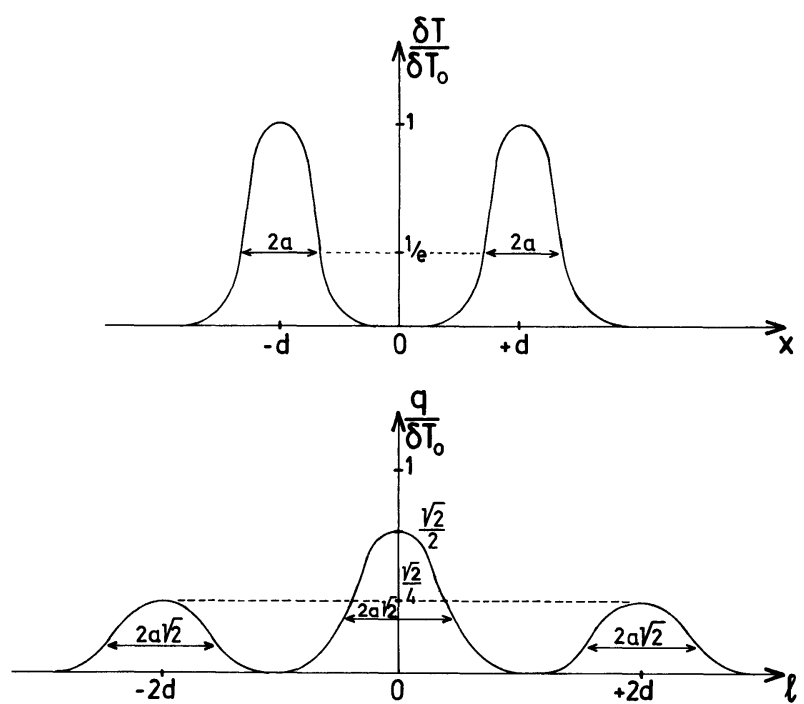

Fig. 2. - Fonction de Richardson $q$ calculée à partir d'une perturbation $\delta T$ correspondant à l'échauffement du liquide par deux faisceaux gaussiens de lumière, de largeur $2 a$ et centrés en $x= \pm d$.

[Richardson function $q(l)$ corresponding to a temperature profile $\delta T(x)$ created by two Gaussian laser beams of width $2 a$ and distant of $2 d$.]

Guidé par la forme de l'équation de Fick, Richardson suggère une équation de diffusion pour $q(l)$

$$
\dot{q}=(\partial / \partial l)[D(l) \partial q / \partial l]
$$

où l'augmentation de la diffusion avec la séparation est prise en compte par une diffusivité $D(l)$ dont la forme est discutée dans le paragraphe suivant; un argument simple permet de voir pourquoi $D(l)$ augmente avec $l$ : l'augmentation au cours du temps de la séparation $l$ entre deux particules est due essentiellement à l'effet des tourbillons de taille inférieure ou égale à $l$; le domaine de taille de tourbillons contribuant à leur séparation sera donc d'autant plus grand qu'elles seront plus éloignées.

Richardson a montré [7] qu'une équation de Fick généralisée de la forme linéaire suivante

$$
\dot{T}(x, t)=\sum_{\mathrm{r}, \mathrm{s}} K_{\mathrm{rs}} \partial^{\mathrm{r}+\mathrm{s}} T(x, t) /\left(\partial x^{\mathrm{r}} \partial t^{\mathrm{s}}\right)
$$

où les diffusivités généralisées $K_{\mathrm{rs}}$ seraient indépen- 
dantes de $x$ et de $t$ ne permet pas de rendre compte des expériences. L'argument utilise le fait qu'il n'existe pas de loi de superposition linéaire permettant de décrire l'étalement d'une tache thermique, somme de deux taches thermiques indépendantes.

Par ailleurs, Batchelor [8] a suggéré qu'une équation de la forme

$$
\dot{q}=K(t) \partial^{2} q / \partial x^{2}
$$

pourrait aussi rendre compte des observations expérimentales.

Plus récemment, Kraichman [14] a écrit une équation d'évolution pour $q(l, t)$ :

$$
\dot{q}=(\partial / \partial l)[\eta(l, t) \partial q / \partial l]
$$

où $\eta(l, t)$ représente un coefficient de diffusion qui prend deux formes différentes suivant que le temps $t$ est petit ou grand devant le temps de rotation $l / u(l)$ d'un tourbillon de taille $l$ et de vitesse $u(l)$.

Nous voyons donc que la forme de l'éq. (2), que nous utiliserons par la suite, n'est pas unique.

2. 3 FoRme DE $D(l)$. - Dès son premier article sur le sujet, Richardson utilisant les mesures de séparation de deux particules distantes d'une séparation $l$ variant entre $15 \mathrm{~m}$ et $1000 \mathrm{~km}$ dans l'atmosphère propose une loi de la forme

$$
D(l)=\alpha l^{4 / 3}
$$

où l'exposant $4 / 3$ est posé comme étant le rationnel le plus simple compatible avec le tracé expérimental des résultats en coordonnées log-log.

De nombreuses mesures expérimentales ont, depuis, conforté la forme de cette loi de $D(l)$; une revue peut être trouvée dans la référence [6], p. 556. Les expériences utilisent la mesure de la vitesse de séparation de deux objets en fonction de leur séparation initiale. La grande gamme de distances désirable pour l'établissement de la loi de puissance est obtenue soit par des particules en suspension dans l'atmosphère [4, 9] ou par des objets flottant sur la mer $[10,15]$. Des mesures plus récentes dans l'atmosphère [16], pour de grandes séparations (entre 100 et $1000 \mathrm{~km}$ ) montrent un comportement de $D(l) \sim l^{2}$; la différence avec la loi en $4 / 3$ citée plus haut vient de ce que, à ces échelles, le mouvement de l'atmosphère peut être considéré comme bidimensionnel.

La justification théorique de la loi de Richardson en 4/3 n'a pu être donnée que bien plus tard, après la description par Kolmogorov en 1941 des hypothèses de similitude des écoulements turbulents [11, 12]. Une présentation détaillée en est donnée par Monin et Yaglom [6] (tome 2, p. 551).

Nous en donnons une explication simplifiée. Considérons deux particules distantes de $l$. La séparation de ces particules provient typiquement des tourbillons de taille $l$, les plus grands tourbillons entraînant, dans un mouvement d'ensemble, les deux objets ; la sépa- ration due aux plus petites échelles corrigerait l'effet des tourbillons $l$. Soit $u(l)$ la vitesse caractéristique de ces tourbillons (c'est aussi la variation de la vitesse locale sur la distance $l$ ). Le coefficient de diffusion relative de ces deux particules pourra s'écrire comme la variation du carré de leur distance avec le temps

$$
D(l) \sim\left(\dot{l}^{2}\right)=2 \dot{l} \sim \operatorname{lu}(l) .
$$

La dernière relation repose sur le fait que $u(l)$ représente aussi la vitesse relative de deux particules distantes de $l$.

Par ailleurs, on a, pour chaque échelle $l$, la relation

$$
\varepsilon \sim u^{2}(l) \cdot[u(l) / l]=u^{3}(l) / l
$$

exprimant la densité d'énergie cinétique par unité de volume. $u^{2}(l)$, dans le temps de vie d'un tourbillon $l$, exprimé dimensionnellement par $l / u(l)$. On déduit de (4) et (5)

$$
D(l) \sim \varepsilon^{1 / 3} l^{4 / 3}
$$

en accord avec la forme de Richardson. Notons que la forme (6) n'est valable que dans le domaine inertiel, c'est-à-dire pour des valeurs de $l$ plus petites que les grandes échelles du mouvement $L$ et supérieures à la microéchelle de Kolmogorov $\xi\left(\sim\left(v^{3} / \varepsilon\right)^{1 / 4}\right)$, caractérisant les distances sur lesquelles la dissipation d'énergie par viscosité est importante.

Le coefficient $\alpha$ de l'éq. (3) ( $\alpha$ est noté $\varepsilon$ dans les références originales !) est directement associé au taux de production d'énergie incidente. Dans la détermination expérimentale de la loi d'échelle en $l^{4 / 3}$, il est nécessaire d'utiliser le même écoulement pour différentes séparations. C'est ce que réalisent implicitement les régimes d'écoulement atmosphériques ou océanographiques des expériences citées plus haut.

3. Solution de l'équation de Richardson. 3. 1 Solution GÉNÉRAlE. - L'éq. (2) permet de calculer $q(l, t)$ connaissant le profil à l'instant initial $t=0$, $q(l, 0)$. Utilisant $(3)$ elle s'écrit

$$
\dot{q}(l, t)=\alpha(\partial / \partial l)\left(l^{4 / 3} \partial q / \partial l\right) .
$$

On cherche des solutions de la forme

$$
q(l, t)=\int_{\lambda} B(\lambda) y_{\lambda}(l) \exp (-\lambda t) \mathrm{d} \lambda
$$

ce qui permet de se ramener à une équation pour la variable spatiale $l$

$$
l^{2} d^{2} y_{\lambda}(l) / \mathrm{d} l^{2}+(4 / 3) l \mathrm{~d} y_{\lambda}(l) / \mathrm{d} l+(\lambda / \alpha) l^{2 / 3} y_{\lambda}(l)=0 \text {. }
$$

Nous décrivons dans l'appendice A la solution de l'éq. (7) qui mène à la forme générale suivante

$$
q(l, t)=(\pi \sqrt{\alpha})^{-1} \int_{0}^{\infty} N(l, m, t) q_{0}(m) \mathrm{d} m
$$


utilisant un noyau intégral, indépendant de la forme du profil $q_{0}(m)=q(m, t=0)$,

$$
\begin{aligned}
N(l, m, t)=\int_{0}^{\infty}[\exp (-\lambda t) / \sqrt{\lambda}]\left[\sin \left(\beta l^{1 / 3}\right) / l^{1 / 3}\right] \times \\
\times\left[\sin \left(\beta m^{1 / 3}\right) / m^{1 / 3}\right] \mathrm{d} \lambda .
\end{aligned}
$$

La forme remarquablement simple de la solution (8), qui ne semble pas encore avoir été obtenue, vient de la valeur 4/3 de l'exposant de (3). On peut transformer l'expression (9) pour faire apparaître des combinaisons sans dimension (voir appendice B), ce qui conduit à :

$$
q(l, t)=Q(u, t)=\pi^{-1 / 2} \int_{0}^{\infty} N(u, v) Q_{0}(v, t) v^{2} \mathrm{~d} v
$$

où les nouvelles coordonnées sans dimension $u$ et $v$ sont définies par

$$
u=\left(3 l^{1 / 3}\right) /(2 \sqrt{\alpha t}) \quad \text { et } \quad v=\left(3 m^{1 / 3}\right) /(2 \sqrt{\alpha t}) .
$$

L'existence de ces variables réduites, dont la forme aurait pu être déduite intuitivement de l'équation de départ, permet de faire des correspondances entre des profils différents ayant une même forme réduite du profil initial $Q_{0}(v, t)$. Si on change les échelles de temps d'un problème à l'autre par le facteur $\eta$, il convient alors de changer les échelles de longueur par un facteur $\eta^{3 / 2}$ (par exemple la longueur d'onde dans le cas d'une modulation sinusoïdale de température que nous considérons dans le paragraphe suivant), soit

$$
q(l, t) \equiv q\left(\eta^{-3 / 2} l, \eta^{-1} t\right) .
$$

On peut aussi faire correspondre les lois d'évolution de la fonction $q(l, t)$ dans deux écoulements caractérisés par des dissipations par unités de masse et de temps $\varepsilon_{1}$ et $\varepsilon_{2}=\mu \varepsilon_{1}$. D'après (6), on a $\alpha_{2}=\mu^{1 / 3} \alpha_{1}$ et l'intégrale (10) conservera la même valeur si on change l'échelle des temps de telle façon que $t_{2}=\mu^{-1 / 3} t_{1}$, soit :

$$
q_{2}(l, t)=q_{1}\left(l, \mu^{1 / 3} t\right) .
$$

3.2 Calcul numérique de La SOlution. - a) Fonction de Richardson à l'instant initial. - Nous considérons l'exemple d'un profil sinusoïdal initial de température, de pas $p=2 \pi / k$

$$
\delta T(x)=\delta T_{0}(1+\cos k x) .
$$

Ce profil correspond au cas de l'expérience actuellement réalisée dans un écoulement laminaire et que nous poursuivons dans le cas d'écoulements turbulents. Il lui correspond un profil

$$
q_{0}(l)=\delta T_{0}(1+(1 / 2) \cos k l)
$$

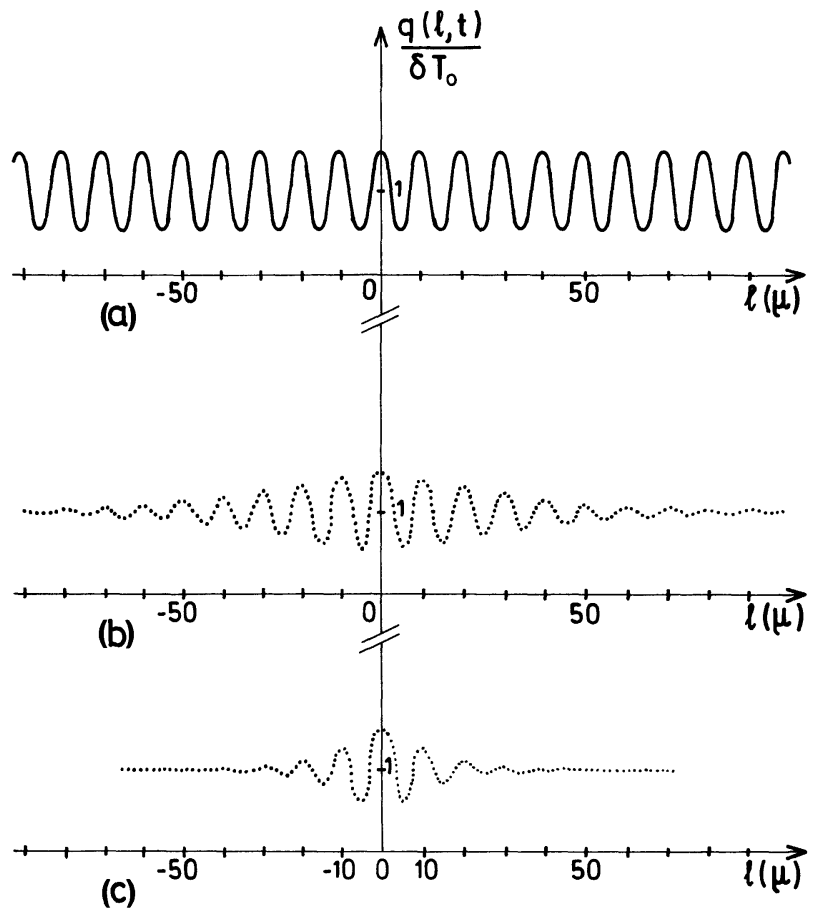

Fig. 3. - Partant du profil sinusoïdal de température (formule (13a), pas $p=10 \mu$ ), la fonction de Richardson a été calculée pour les temps $t=0(a), 100 \mu \mathrm{s}(b), 400 \mu \mathrm{s}(c)$.

[Starting from a sinusoidal temperature profile (formule (13a), period $p=10 \mu$ ) the Richardson function has been calculated for the times $t=0(a), 100 \mu \mathrm{s}(b), 400 \mu \mathrm{s}(c)$.]

représenté sur la figure $3 a$. De façon générale, si le profil initial de température $\delta T(x)$ peut être décomposé en série de Fourier

$$
\delta T(x)=A_{0}+\sum_{n=1}^{\infty}\left(A_{n} \cos n k x+B_{n} \sin n k x\right)
$$

le profil $q(l)$ se met sous la forme [4]

$$
q(l)=A_{0}+1 /\left(2 A_{0}\right) \sum_{n=1}^{\infty}\left[\left(A_{n}^{2}+B_{n}^{2}\right) \cos n k l\right] .
$$

b) Variation dans le temps $q(l, t)$. - Le calcul numérique de l'intégrale (10) pour le profil (13) est donné dans l'appendice $B$ et est représenté sur la figure 3 sous la forme d'une variation de $q(l)$ pour les trois temps $t=0, t=100 \mu \mathrm{s}, t=400 \mu \mathrm{s}$. On a utilisé la valeur de $\alpha=0,4 \mathrm{~cm}^{2 / 3} \mathrm{~s}^{-1}$ donnée dans le travail original de Richardson. On observe pour des temps croissants un grignotement progressif de $q(l)$ par les bords qui est dû à l'effet plus rapide de la diffusion pour les grandes séparations. Par contre, la forme de la variation sinusoïdale pour les petites séparations $l \sim p$ est conservée sur des plus grandes échelles de temps. Dans le cas d'une diffusion fickienne avec une diffusivité thermique $\kappa$ constante l'atténuation de l'amplitude des oscillations de $q(l, t)$ se ferait, de façon indépendante de $l$, avec une constante de temps de décroissance exponentielle :

$$
\left(2 K k^{2}\right)^{-1} \text {. }
$$


4. Utilisation de $q(l)$; diffraction. - 4.1 CALCUL DE L'INTENSITÉ DIFFRACTÉE. - L'étude expérimentale que nous développons actuellement utilise la figure de la diffraction de la lumière par la perturbation de température. Cette figure peut être déduite très directement de la fonction $q(l, t)$. En effet, l'amplitude diffractée avec un vecteur d'onde $\gamma=\mathbf{k}_{\mathrm{d}}-\mathbf{k}_{\mathrm{i}}$ (i et d se rapportent au vecteur incident et diffracté), s'écrit

$$
A_{\mathrm{d}}(\gamma)=\int(\delta n / \delta T) \delta T(x) \exp (i \gamma x) \mathrm{d} x
$$

d'où l'intensité diffractée

$$
\begin{aligned}
\mathfrak{J}_{\mathrm{d}}(\gamma)= & A_{\mathrm{d}}(\gamma) A_{\mathrm{d}}^{*}(\gamma)=(\delta n / \delta T)^{2} \int \delta T(x) \times \\
& \quad \times \exp (i \gamma x) \mathrm{d} x \int \delta T(y) \exp (-i \gamma y) \mathrm{d} y \\
= & (\delta n / \delta T)^{2} \iint \delta T(x) \delta T(y) \exp [i \gamma(x-y)] \mathrm{d} x \mathrm{~d} y \\
= & (\delta n / \delta T)^{2} \int q(l) \exp (i \gamma l) \mathrm{d} l= \\
& =(\delta n / \delta T)^{2} I_{\mathrm{d}}(\gamma, t) .
\end{aligned}
$$

Le calcul d'intensité se ramène directement à celui de la transformée de Fourier de $q(l)$.

La figure 4 présente les résultats de ce calcul (effectué numériquement par décomposition en série de

(a)

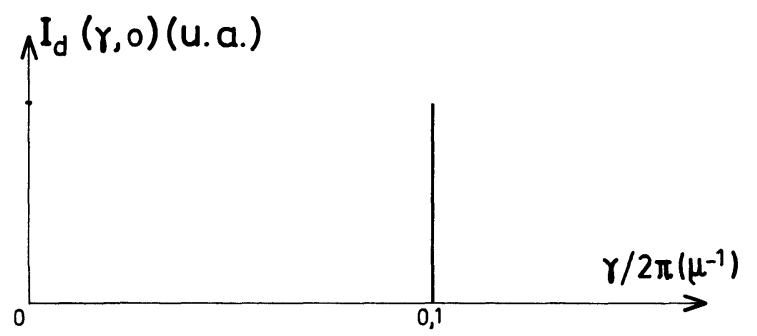

(b)

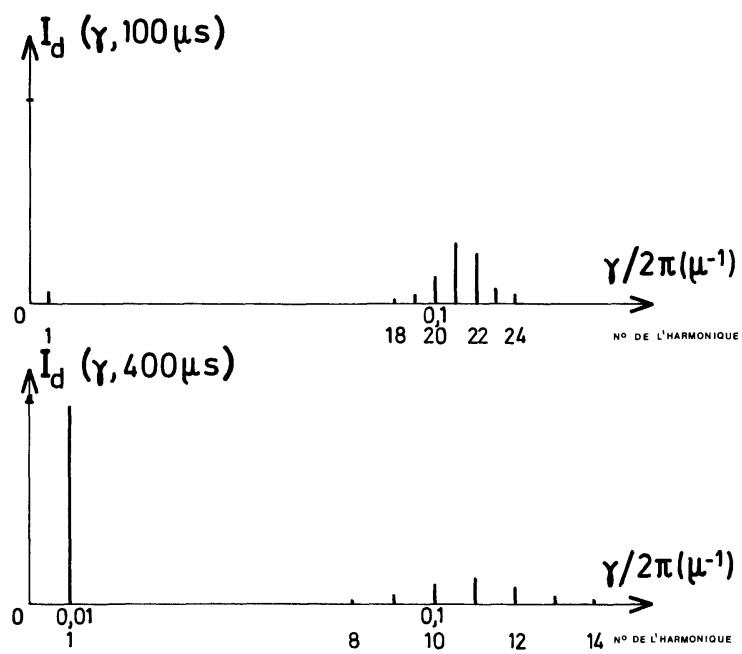

Fig. 4. - Etalement de l'intensité diffractée autour du pic de Bragg $\left(\gamma / 2 \pi=0,1 \mu^{-1}\right)$. Les 3 courbes correspondent à la figure 3 .

[Spread of the diffracted intensity around the Bragg peak $\left(\gamma / 2 \pi=0.1 \mu^{-1}\right)$ for the profiles of figure 3.]
Fourier) pour les profils et les différents temps de calcul correspondant à la figure 3 . On constate que le pic de Dirac dû au réseau sinusoïdal initial s'étale lorsque le temps croît et que le profil $q(l)$ devient moins large. La période fondamentale (grande devant le pas du réseau et l'extension de la figure pour des temps finis) est celle de la répétition du motif $q(l)$, le calcul d'intégrale de Fourier se ramenant à une série de Fourier avec cette grande période (l'utilisation d'une période de répétition plus longue permettrait une description plus continue du profil de la courbe d'intensité diffractée sans en modifier la forme générale).

4.2 DÉTERMination DiReCTE. - On peut obtenir une forme analytique approchée de la fonction $q(l, t)$ dans la limite où l'intervalle de temps entre écriture et lecture de la tache thermique est suffisamment court pour que les effets de diffusion turbulente ne se fassent pas sentir sur les échelles $l \sim p$.

Dans ces conditions, nous écrivons $q(l, t)$ comme le produit d'une variation sinusoïdale $\exp (i k l)$ par une fonction enveloppe lentement variable $f(l, t)$ de telle façon que la relation (2) s'écrive

$$
[\exp (i k l)] \dot{f}=(\partial / \partial l)[D(l)(\partial / \partial l)(\exp (i k l) f(l, t))] .
$$

Utilisant le fait que $|\partial f / \partial l| \ll|i k f|$, on a :

$$
[\exp (i k l)] \dot{f} \sim-k^{2} D(l)[\exp (i k l)] f
$$

dont la solution est

d'où

$$
\begin{aligned}
f & =A \exp \left[-D(l) k^{2} t\right] \\
q(l, t) & =A \exp \left[i k l-\alpha k^{2} l^{4 / 3} t\right] .
\end{aligned}
$$

Aux temps courts, la fonction de Richardson est une sinusoïde enveloppée par une fonction exponentielle.

Nous avons comparé les résultats du calcul numérique direct, et de la forme approchée (15) dans les conditions de la figure 2. L'accord entre les deux formes est excellent (Fig. 5). Le coefficient de corrélation de la régression linéaire (nombre compris entre 0 et 1 )

(a)

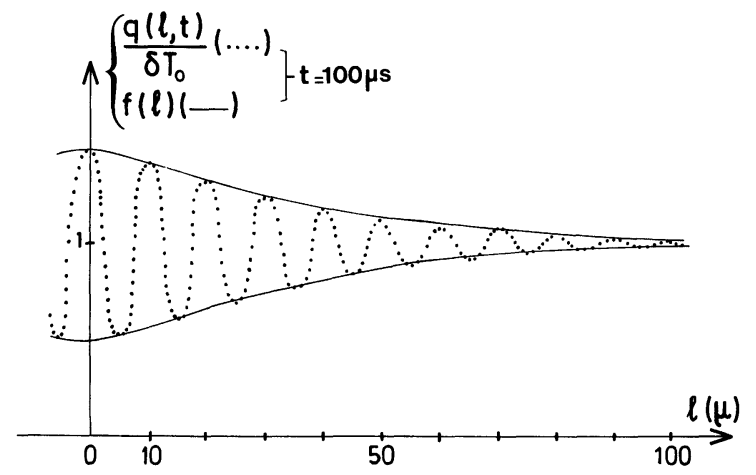

Fig. 5. - Comparaison entre la fonction calculée (Fig. $3 b$ ) et la solution enveloppe approchée (formule (15a)).

[Comparison between the calculated function (Fig. $3 b$ ) and the approximate solution (formula (15a)).] 
entre les valeurs du Logarithme de l'enveloppe, que l'on prend au niveau des entre-extremums de la fonction $q(l, t)$ et $l^{4 / 3}$ est de 0,99 pour les temps de 100 et $400 \mu$ s (variations des figures $2 b$ et $c$ ).

Nous pouvons maintenant préciser la condition temps courts de validité de l'expression $t<t_{1}$ en exprimant que la largeur $l_{1}$ de l'enveloppe (évaluée à $1 / e$ de la valeur à l'origine) est suffisamment grande devant le pas de la sinusoïde, soit

$$
k l_{1} \gg 1 \text { avec } l_{1} \text { défini par } D\left(l_{1}\right) k^{2} t_{1}=1 .
$$

On peut calculer l'intensité diffractée à partir de cette expression, soit :

$$
\begin{aligned}
I_{\mathrm{d}}(\gamma, t)=\int & q(l, t) \exp (-i \gamma l) \mathrm{d} l \propto \\
& \propto \int \exp \left[i(k-\gamma) l-D(l) k^{2} t\right] \mathrm{d} l .
\end{aligned}
$$

Cette expression indique l'existence du pic de diffraction de vecteur d'onde $\gamma \sim k$ et de largeur $\Delta \gamma \sim 2 \pi l_{1}^{-1}$ où $l_{1}(t)$ est défini par $D\left(l_{1}\right) k^{2} t \sim 1$. Ce résultat est en accord avec les résultats du calcul de transformée de Fourier de la figure 4.

5. Problème à trois dimensions. - 5.1 Equation DE RICHARDSON. - On considère une perturbation tridimensionnelle de température caractérisée par un profil $\delta T(\mathbf{r}, t)$ et l'analogue tridimensionnel de $q(l, t)$, qui est la fonction de corrélation de la température par unité de volume et pour les couples de points $\mathbf{r}$ et $\mathbf{r}+\mathbf{s}, q(\mathbf{s}, t)$. L'équation de Richardson se généralise en

$$
\dot{q}(\mathbf{s}, t)=\nabla[D(|\mathbf{s}|) \nabla[q(\mathbf{s}, t)]] .
$$

Dans un cas de diffusion turbulente isotrope, $D$ ne dépend que de la distance $s=|\mathbf{s}|$ entre deux points.

Regardons la forme de l'équation dans le problème tridimensionnel de la diffusion d'une grille thermique sinusoïdale de température (Fig. 6). Utilisant les coordonnées cylindriques, définies dans la figure, compatibles avec la symétrie du problème

$$
\begin{aligned}
\dot{q}=(\partial / \partial l)[D(|\mathbf{s}|) \partial q / \partial l] & + \\
& +D(|\mathbf{s}|)(1 / \rho)(\partial / \partial \rho)(\rho \partial q / \partial \rho) \\
& +2 \rho\left(\rho^{2}+l^{2}\right)^{-1 / 3} \partial q / \partial l .
\end{aligned}
$$

En particulier, le long de l'axe de $l$ perpendiculaire aux plans isothermes, on a :

$$
\begin{aligned}
\dot{q}=(\partial / \partial l)[D(l) \partial q / \partial l] & \\
& +D(l) \lim _{\rho \rightarrow 0}[(1 / \rho)(\partial / \partial \rho)(\rho \partial q / \partial \rho)] .
\end{aligned}
$$

Le second terme, qui ne peut pas être exprimé simplement en fonction de $q$, ne peut être négligé. On voit que le problème tridimensionnel ne se réduit

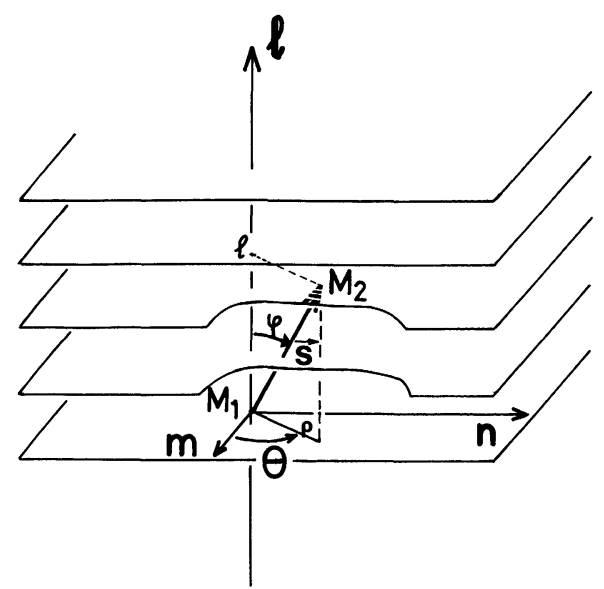

Fig. 6. - Grille thermique dans le problème à trois dimensions. L'importance des transferts pour des vecteurs $\mathbf{s}$ obliques ne permet pas de ramener le problème le long de l'axe $l$ au problème à 1 dimension.

[Thermal grid in a 3 dimensional problem. The importance of transfers with oblique $\mathbf{s}$ vectors is such that the problem does not reduce to a 1 dimensional problem for the diffusion along the axis $l$.]

pas au problème à une dimension pour lequel seul le premier terme du second membre existe. Le terme supplémentaire traduit le fait que, dans la diffusion à trois dimensions, les transferts obliques (par rapport à l'axe $l$ ) de chaleur ne peuvent être négligés.

5.2 FONCTION DE RICHARDSON ET INTENSITÉ DIFFRACTÉE. - Nous n'avons pas obtenu, dans ce problème à 3 dimensions, de représentation intégrale de la solution de l'éq. (17), mais nous avons directement généralisé les considérations données au paragraphe IV . 2 pour obtenir la solution aux temps courts : le segment de longueur $l$, peu affecté par la diffusion au temps $t$ est remplacé dans ce cas par une sphère de rayon $R(t)$ tel que : $D(R) k^{2} t=1$, la condition temps courts s'exprimant par $k R(t) \gg 1$. La solution approchée s'écrit :

$$
q(\mathbf{s}, t)=A \exp \left[i \mathbf{k} . \mathbf{s}-D(s) k^{2} t\right] .
$$

Remarquons que cette expression ne convient pas pour les valeurs de $s$ telles que $D(s)<2 \kappa$ (le coefficient $D$ ne peut en effet devenir inférieur à la diffusivité thermique moléculaire-relative $2 \kappa$ ); nous écrirons donc en première approximation :

$$
q(\mathbf{s}, t)=A \exp \left[i \mathbf{k} . \mathbf{s}-D(s) k^{2} t\right] \exp \left(-2 \kappa k^{2} t\right) .
$$

L'intensité diffractée s'écrit alors :

$$
\begin{aligned}
I_{\mathrm{d}}(\gamma, t)=A \exp \left(-2 \kappa k^{2} t\right) \int & \exp [i(\mathbf{k}-\gamma) \cdot \mathbf{s}- \\
& \left.-D(s) k^{2} t\right] \mathrm{d} \mathbf{s} .
\end{aligned}
$$


Le calcul de cette expression est donné dans l'appendice $\mathrm{C}$ [17] et conduit à :

$$
\begin{aligned}
I_{\mathrm{d}}(\gamma, t) \sim \exp \left(-2 \kappa k^{2} t\right)\left[\left(\alpha k^{2} t\right)^{-9 / 4}-\right. \\
\left.-0,7\left(\alpha k^{2} t\right)^{-15 / 4}(k-\gamma)^{2}\right] .
\end{aligned}
$$

Nous obtenons ainsi une variation parabolique en $(k-\gamma)$ de l'intensité diffractée dans l'ordre (1), représentée sur la figure 7 ; la largeur à mi-hauteur de ce pic s'écrit :

$$
\Delta \gamma \sim 1,8\left(\alpha k^{2} t\right)^{3 / 4}=1,8[R(t)]^{-1}
$$

et l'intensité au centre du pic vaut :

$$
\begin{aligned}
I_{\mathrm{d}}(\gamma=\boldsymbol{k}, t) \sim \exp \left(-2 \kappa k^{2} t\right) & \left(\alpha k^{2} t\right)^{-3 / 4}= \\
= & R^{3}(t) \exp \left(-2 \kappa k^{2} t\right) .
\end{aligned}
$$

(a)

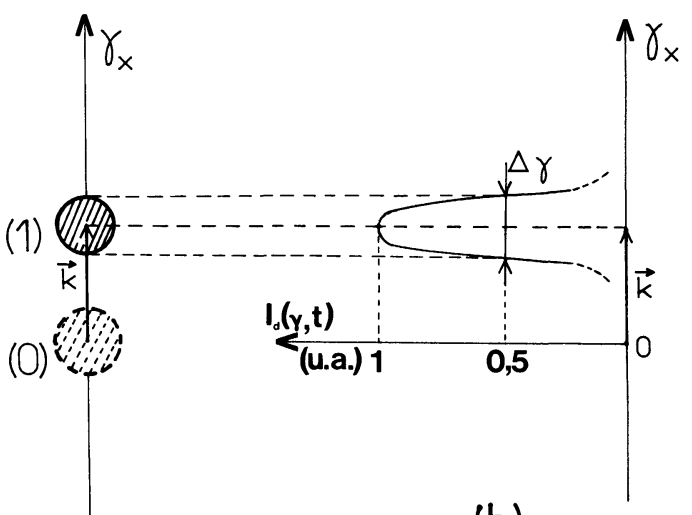

Fig. 7. - (a) Représentation du plan image de Fourier ; disposition de l'ordre 1 diffracté : tache de lumière centrée en $\gamma=\mathbf{k}$ et de largeur $|\Delta \gamma| \sim R^{-1}, R$ étant la taille de la zone du réseau n'ayant pas diffusé. (b) Forme de l'intensité diffractée dans l'ordre (1). La courbe en pointillés indique la zone où cette intensité n'a pas été calculée.

[(a) In the Fourier image plane the first diffracted spot is centered at $\gamma=\mathbf{k}$ and has a width $|\Delta \gamma| \sim R^{-1}$, where $R$ is the size of the grating not affected by diffusion. (b) Shape of the diffracted intensity in the first spot; the dashed curve indicates a region where the intensity has not been calculated.]

Note. - Précisons la relation entre la discussion théorique que nous venons de donner et les expériences entreprises en régime turbulent et qui utilisent l'appareillage décrit en introduction. A la suite de chaque marquage thermique par le laser pulsé, la grille est convectée de façon déterministe par l'écoulement turbulent, de même que chaque observation de Richardson décrivant par exemple une expérience de séparation de deux ballons lâchés à un temps initial. De fait, la lecture, par diffraction du second laser, de la grille convectée pour une petite surface de celle-ci et sur un court intervalle de temps montre des larges fluctuations d'un tir laser à l'autre, tant sur les gradients moyens que sur l'intensité dans les taches. Afin de réaliser les moyennes qu'implique le traitement statistique de la turbulence, plusieurs approches expéri- mentales sont en principe possibles : allonger le temps (ce qui n'est guère possible vue la loi de décroissance de l'intensité diffractée plus rapide en régime turbulent que laminaire), augmenter la surface de lecture, ou encore utiliser des moyennes sur un grand nombre de données. C'est cette dernière solution que nous avons retenue, en utilisant les facilités d'une chaîne d'acquisition de données optiques couplée à un analyseur multicanaux. L'équivalence entre ces divers types de mesure nous semble relever du même type de problèmes que l'hypothèse ergodique en mécanique statistique.

6. Conclusion. - L'analyse modèle que nous avons donnée de la diffusion turbulente de paires de particules décrite à partir de la fonction de Richardson peut être vérifiée par étude de l'intensité diffractée (expression (21)) dans l'expérience de la figure 1. Donnons quelques caractéristiques d'une expérience dans un écoulement turbulent (produit par exemple en introduisant une grille de barreaux espacés de $L \sim 1 \mathrm{~mm}$ ). Prenant une amplitude de fluctuation turbulente de vitesse $u(L) \sim 10^{-2} \mathrm{~cm} / \mathrm{s}$, nous obtenons un coefficient $\alpha$ pour la loi de Richardson $\alpha \sim \varepsilon^{1 / 3} \sim\left(u^{3} / L\right)^{1 / 3}=10 \mathrm{~cm}^{2 / 3} \mathrm{~s}^{-1}$ (voir formule (6)). Ce coefficient est 20 fois plus grand que le coefficient utilisé dans les calculs de cet article (il est possible de changer d'échelle de $\alpha$ à l'aide de la formule (12)): la différence est due au fait que la turbulence utilisée par Richardson est celle produite aux plus grands nombres de Reynolds des mouvements atmosphériques. Dans le cas de notre expérience, on peut déduire que les échelles de temps typiques d'évolution du réseau initial sont de l'ordre de quelques $10^{-5} \mathrm{~s}$, valeur qui reste accessible expérimentalement. Par une étude du profil de la tache de diffraction produite en fonction du délai $\Delta t$, il doit être possible de vérifier directement la forme de la loi de Richardson.

Dans son article original, de Gennes a aussi suggéré la mesure de l'étalement d'une tache ponctuelle pour l'étude de la diffusion de paires de particules. Pour ce problème que nous n'avons pas discuté, la fonction $q(l)$ est connue analytiquement. Un tel exemple permet de faire les remarques suivantes :

- d'une part, elle permet de souligner la différence - implicite le long de cet article - entre la diffusion de paires de particules discutée ici et le simple étalement d'une tache thermique tel que le mesurerait une des techniques interférométriques largement utilisées dans l'étude des écoulements hydrodynamiques,

- d'autre part, elle montre l'intérêt de l'étude à partir de grille - comparable à celui de la technique de Rayleigh forcé [5] - dans des systèmes au repos qui est de pouvoir sélectionner des longueurs d'onde privilégiées d'un problème particulier, en relation, dans le cas présent, avec les échelles de la turbulence. De plus, le réseau présente l'avantage par rapport au point chaud de réaliser déjà une première moyenne (de donner des signaux de diffraction plus intenses!). 
Notons enfin que nous étudions la possibilité d'une visualisation directe de la grille thermique formée dans l'écoulement, en utilisant une méthode optique du type holographie ou speckle; une telle expérience permettrait de mettre en évidence des effets locaux (à l'échelle du pas de la grille) qui ne sont pas vus dans l'analyse par diffraction (par suite du moyennage sur l'étendue du faisceau de diffraction); une des principales difficultés d'une telle expérience est le caractère tridimensionnel du champ de vitesses turbulent.
Remerciements. - Ce travail doit énormément à des discussions avec P. G. de Gennes. Nous devons aussi beaucoup à $\mathrm{M}$. Fermigier et $\mathrm{P}$. Jenffer avec qui nous développons ces expériences. Nous tenons aussi à remercier U. Frisch, R. Moreau, P. Pieranski, E. Siggia et W. Webb pour des échanges sur ce thème. Nous sommes redevables à X. Guyon et J. M. Bony pour une discussion sur la solution de l'équation de Richardson.

\section{APPENDICE A}

Détermination de la solution générale de l'équation différentielle :

$$
l^{2} d^{2} y_{\lambda}(l) / \mathrm{d} l^{2}+(4 / 3) l d y_{\lambda}(l) / \mathrm{d} l+(\lambda / x) l^{2 / 3} y_{\lambda}(l)=0
$$

que l'on écrit sous forme simplifiée

$$
l^{2} y^{\prime \prime}+(4 / 3) l y^{\prime}+(\lambda / \alpha) l^{2 / 3} y=0
$$

équation de la forme :

$$
l^{2} y^{\prime \prime}+(2 a+1) l y^{\prime}+\left(b^{2} l^{2 m}+c^{2}\right) y=0
$$

dont la solution générale est :

$$
y=l^{-a} Z_{k / m}\left(b l^{m} / m\right)
$$

où $k=\sqrt{a^{2}-c^{2}}$ et $Z_{k / m}$ est une combinaison linéaire des fonctions de Bessel d'ordre $k / m$; nous obtenons ici :

avec

$$
y=l^{-1 / 6} Z_{1 / 2}\left(3 \sqrt{\lambda / \alpha} l^{1 / 3}\right)
$$

$$
Z_{1 / 2}(L)=C_{1} J_{1 / 2}(L)-C_{2} J_{-1 / 2}(L) .
$$

Or, à l'origine la fonction $J_{1 / 2}$ est régulière mais $J_{-1 / 2}$ ne l'est pas ; la fonction $y(l)$ devant le rester, nous gardons :

Soit :

$$
y=l^{-1 / 6} C_{1} J_{1 / 2}\left(3 \sqrt{\lambda / \alpha} l^{1 / 3}\right) .
$$

$$
y=C_{1} \sqrt{2 / 3 \pi}(\alpha / \lambda)^{1 / 4} l^{-1 / 3} \sin \left(3 \sqrt{\lambda / \alpha} l^{1 / 3}\right) \text {. }
$$

D’où la solution générale :

$$
q(l, t)=\int_{\lambda} B(\lambda) y_{\lambda}(l) \exp (-\lambda t) \mathrm{d} \lambda
$$

la constante $B(\lambda)$ étant déterminée par le profil initial $q(l, 0)$, soit par l'équation

$$
q(l, 0) \equiv q_{0}(l)=\int_{\lambda} B(\lambda) y_{\lambda}(l) \mathrm{d} \lambda .
$$

En effectuant le changement de variables :

$$
z=\frac{3 l^{1 / 3}}{\sqrt{\alpha}} \text { et } \mu=\sqrt{\lambda}
$$

nous faisons apparaître la transformée de Fourier en sinus de $B(\mu)$, soit :

$$
\int B_{1}(\mu) \sqrt{\mu} \sin (\mu z) \mathrm{d} \mu=\left(1 / 2 C_{1}\right) \sqrt{\pi / 6} \alpha^{1 / 4} z r_{0}(z)
$$

avec $r_{0}(z)=q_{0}\left(z^{3}\right)$. 
Donc, par transformation inverse

$$
\sqrt{\mu} B_{1}(\mu)=1 /\left(C_{1} \sqrt{6 \pi}\right) \alpha^{1 / 4} \int \sin (\mu z) z r_{0}(z) \mathrm{d} z
$$

Revenant aux variables $l$ et $\lambda$ et posant $\beta=3 \sqrt{\lambda / \alpha}$, on obtient finalement :

$$
B(\lambda)=\left(1 / C_{1}\right) \sqrt{3 / 2 \pi} \alpha^{-3 / 4} \lambda^{-1 / 4} \int q_{0}(l) l^{-1 / 3} \sin \left(\beta l^{1 / 3}\right) \mathrm{d} l .
$$

L'expression générale de $q(l, t)$ est donc

$$
\begin{aligned}
q(l, t) & =(\pi \sqrt{x})^{-1} \int_{\lambda}(1 / \sqrt{\lambda})\left[\int q_{0}(m) m^{-1 / 3} \sin \left(\beta m^{1 / 3}\right) \mathrm{d} m\right] l^{-1 / 3} \sin \left(\beta l^{1 / 3}\right) \exp (-\lambda t) \mathrm{d} \lambda \\
& =(\pi \sqrt{x})^{-1} \int_{0}^{\infty}\left[\int_{\lambda}[\exp (-\lambda t) / \sqrt{\lambda}]\left[\sin \left(\beta l^{1 / 3}\right) / l^{1 / 3}\right]\left[\sin \left(\beta m^{1 / 3}\right) / m^{1 / 3}\right] \mathrm{d} \lambda\right] q_{0}(m) \mathrm{d} m
\end{aligned}
$$

ce qui peut s'écrire :

$$
q(l, t)=(\pi \sqrt{\alpha})^{-1} \int_{0}^{\infty} N(l, m, t) q_{0}(m) \mathrm{d} m
$$

avec :

$$
N(l, m, t)=\int_{0}^{\infty}[\exp (-\lambda t) / \sqrt{\lambda}]\left[\sin \left(\beta l^{1 / 3}\right) / l^{1 / 3}\right]\left[\sin \left(\beta m^{1 / 3}\right) / m^{1 / 3}\right] \mathrm{d} \lambda
$$

\section{APPENDICE B}

Calcul numérique de la fonction de Richardson. - B1. TRANSFORMATION DE L'EXPRESSION (9) POUR ARRIVER A UNE EXPRESSION NE COMPORTANT QUE DES COMBINAISONS ADIMENSIONNELLES (EXPRESSION (10)). Nous partons de

soit :

$$
N(l, m, t)=\int_{0}^{\infty}[\exp (-\lambda t) / \sqrt{\lambda}]\left[\sin \left(\beta l^{1 / 3}\right) / l^{1 / 3}\right]\left[\sin \left(\beta m^{1 / 3}\right) / m^{1 / 3}\right] \mathrm{d} \lambda
$$

$$
N(l, m, t)=1 /\left(2 l^{1 / 3} m^{1 / 3}\right) \int_{0}^{\infty}[\exp (-\lambda t) / \sqrt{\lambda}]\left\{\cos \left[\beta\left(l^{1 / 3}-m^{1 / 3}\right)\right]-\cos \left[\beta\left(l^{1 / 3}+m^{1 / 3}\right)\right]\right\} \mathrm{d} \lambda
$$

Par transformation et en posant $\mu=\sqrt{\lambda}$ et $z_{ \pm}=3 / \sqrt{\alpha}\left(l^{1 / 3} \pm m^{1 / 3}\right)$, on obtient :

soit :

$$
N(l, m, t)=1 /\left(l^{1 / 3} m^{1 / 3}\right) \int_{0}^{\infty} \exp \left(-t \mu^{2}\right)\left[\cos \left(\mu z_{-}\right)-\cos \left(\mu z_{+}\right)\right] \mathrm{d} \mu
$$

$$
N(l, m, t)=\sqrt{\pi} /\left(2 l^{1 / 3} m^{1 / 3} \sqrt{t}\right)\left[\exp \left\{-9 /(4 \alpha t)\left(l^{1 / 3}-m^{1 / 3}\right)^{2}\right\}-\exp \left\{-9 /(4 \alpha t)\left(l^{1 / 3}+m^{1 / 3}\right)^{2}\right\}\right]
$$

car les intégrales du type $\int_{0}^{\infty} \mathrm{e}^{-t \mu^{2}} \cos (\mu z) \mathrm{d} \mu$ valent $\frac{\sqrt{\pi}}{2 \sqrt{t}} \mathrm{e}^{-z^{2} / 4 t}$.

D’où :

$$
q(l, t)=Q(u, t)=(\pi)^{-1 / 2} \int_{0}^{\infty} 1 /(u v)\left[\exp \left\{-(u-v)^{2}\right\}-\exp \left\{-(u+v)^{2}\right\}\right] Q_{0}(v, t) v^{2} \mathrm{~d} v
$$
soit :

$$
q(l, t)=Q(u, t)=(\pi)^{-1 / 2} \int_{0}^{\infty} N(u, v) Q_{0}(v, t) v^{2} \mathrm{~d} v
$$

avec :

$$
N(u, v)=1 /(u v)\left[\exp \left\{-(u-v)^{2}\right\}-\exp \left\{-(u+v)^{2}\right\}\right]
$$


B2. CAlCUl NUMÉRIQUE DE L'EXPRESSION (10). - Nous avons calculé numériquement cette intégrale par la méthode des trapèzes en prenant un pas d'intégration petit devant celui du réseau initial (ici il est de l'ordre de $p / 60)$; les bornes d'intégration ont été choisies en fonction de la décroissance assez rapide de la fonction $N(u, v)$ : en effet, il s'agit d'une forme gaussienne de largeur typique 2 unités qui ne prend donc plus de valeurs appréciables en dehors de l'intervalle $[u-2, u+3]$, à $u$ fixé ; par ailleurs, son amplitude décroît assez rapidement avec $u$, ce qui confirme qualitativement que les valeurs de l'intégrale (c'est-à-dire $q(l, t)$ ) diminueront lorsque augmentera la séparation (qui correspond aux grandes valeurs de $u$ ).

\section{APPENDICE C}

Calcul analytique de l'intensité diffractée dans le problème à 3 dimensions. - Nous partons de :

$$
I_{\mathrm{d}}(\gamma, t)=A \exp \left(-2 \kappa k^{2} t\right) \int \exp \left[i(\mathbf{k}-\gamma) . \mathbf{s}-D(s) k^{2} t\right] \mathrm{d} \mathbf{s} .
$$

Posant $\zeta=\mathbf{k}-\boldsymbol{\gamma}$ dans cette expression et ne considérant que les directions de $\zeta$ suivant $\mathbf{k}$, nous obtenons :

$$
I_{\mathrm{d}}(\zeta, t)=A \exp \left(-2 \kappa k^{2} t\right) \iiint \exp [i \zeta \cos \varphi] \exp \left[-D(s) k^{2} t\right] s^{2} \mathrm{~d} s \sin \varphi \mathrm{d} \theta \mathrm{d} \varphi
$$

où les variables $\theta$ et $\varphi$ sont définies sur la figure 6 ; par intégration sur ces variables, nous obtenons :

$$
I_{\mathrm{d}}(\zeta, t)=A \exp \left(-2 \kappa k^{2} t\right) \int_{0}^{\infty} 4 \pi s^{2}[\sin (\zeta s) / \zeta s] \exp \left(-D(s) k^{2} t\right) \mathrm{d} s
$$

Un développement limité de $\sin (\zeta s)$ conduit à :

$$
I_{\mathrm{d}}(\zeta, t) \simeq A(4 \pi) \exp \left(-2 \kappa k^{2} t\right) \int_{0}^{\infty}\left(s^{2} / \zeta\right)\left(\zeta s-\zeta^{3} s^{3} / 6\right) \exp \left(-\alpha s^{4 / 3} k^{2} t\right) \mathrm{d} s / s .
$$

Nous nous intéressons en effet à l'intensité diffractée dans l'ordre 1, c'est-à-dire au voisinage de $\boldsymbol{\gamma}=\boldsymbol{k}(\boldsymbol{\zeta}=0)$. Posant : $T=\alpha k^{2} t$ et $y=T s^{4 / 3}$, il vient (au facteur multiplicatif $3 \pi A$ près) :

$$
\begin{aligned}
I_{\mathrm{d}}(\zeta, T) & \sim \exp \left(-2 \kappa k^{2} t\right) \int_{0}^{\infty}\left[T^{-9 / 4} y^{9 / 4}-\left(\zeta^{2} / 6\right) T^{-15 / 4} y^{15 / 4}\right] \exp (-y) \mathrm{d} y \\
& \sim \exp \left(-2 \kappa k^{2} t\right)\left[T^{-9 / 4} \Gamma(9 / 4)-\left(\zeta^{2} / 6\right) T^{-15 / 4} \Gamma(15 / 4)\right]
\end{aligned}
$$

où la fonction $\Gamma(u)$ est définie par : $\Gamma(u)=\int_{0}^{\infty} y^{u-1} \exp (-y) \mathrm{d} y$.

Soit, en revenant aux variables $t$ et $\gamma$ :

$$
I_{\mathrm{d}}(\gamma, t) \sim \exp \left(-2 \kappa k^{2} t\right)\left[\left(\alpha k^{2} t\right)^{-9 / 4}-0,7\left(\alpha k^{2} t\right)^{-15 / 4}(k-\gamma)^{2}\right]
$$

au $4^{\mathrm{e}}$ ordre près en $(k-\gamma)$.

\section{Bibliographie}

[1] Ces expériences actuellement en cours à l'Université de Cornell (WEBB, W.) ne sont pas publiées sauf en partie dans une thèse de PhD (Johnson, D.) 1975.

[2] De Gennes, P. G., J. Phys. Lett. 38 (1977) L 1.

[3] Fermigier, M., Jenffer, P., Petit, L. et GuYon, E., à paraître.

[4] Richardson, L. F., Proc. R. Soc. A 110 (1926) 709.

[5] Rondelez, F., Hervet, H., Urbach, W., Chem. Phys. Lett. 53 (1978) 138.

[6] Monin, A. S. \& Yaglom, A. M., Statistical fluid mechanics 2 (M.I.T. Press) 1975.

[7] Richardson, L. F., Proc. R. Soc. A 214 (1952) 1.

[8] Batchelor, G. K., Proc. Cambridge Philos. Soc. 48 (1952a) 345 .
[9] Richardson, L. F., Beitr. Physiol. freien Atmosph. 15 (1929) 24.

[10] Richardson, L. F. \& Stommel, H., J. Sci. Meteorol. 5 (1948) 238.

[11] Weizsäcker, C. V. von, Z. Phys. 124 (1948) 614.

[12] Batchelor, G. K., Q. J. R. Meteorol. Soc. 76 (1950) 133.

[13] Van Kampen, N. G., Phys. Rep. Phys. Lett. C 24C (1976) 171.

[14] Kraichman, R. H., Phys. Fluids 9 (1966) 1937.

[15] STOMmel, H., J. Mar. Res. 8 (1949) 199.

[16] Morel, P. et Larchevêque, M., J. Atm. Sci. 31 (1974) 2189.

[17] Nous remercions C. MITESCU pour l'aide qu'il nous a apportée dans ce calcul. 